\title{
CARTAN SUBSPACES OF SYMMETRIC LIE ALGEBRAS
}

BY

\author{
J. LEPOWSKY $\left({ }^{1}\right)$ AND G. W. MCCOLLUM
}

\begin{abstract}
A symmetric Lie algebra is defined, following J. Dixmier, to be a Lie algebra $g$ with a decomposition $g=t \oplus p$ such that $\xi$ is a subalgebra of $g,[t, p] \subset p$ and $[p, p] \subset t$. A definition of Cartan subspace of a symmetric Lie algebra is given, and a theory is presented which parallels the standard theory of Cartan subalgebras of Lie algebras, and which generalizes the classical results for real and complex semisimple symmetric Lie algebras.
\end{abstract}

Introduction. In this paper, we first develop a theory of Cartan subspaces of symmetric Lie algebras over infinite fields of any characteristic. Then we specialize to characteristic zero, and finally to reductive Lie algebras over characteristic zero. The results here are suggested by those in $[1, \S 1.13],[4, \S \mathrm{I} .1]$ and [5, §2.5 and Exercises 13-15 at the end of Part 2].

We are indebted to D. Haile for certain results in $\S \S 4$ and 5 .

1. Weight theory for nil sets. In this section, we sketch a theory of weights in a slightly more general setting than the standard one. Throughout this section, $g$ is a Lie algebra over a field $k$ and $\pi: g \rightarrow$ End $V$ is a representation of $g$ on a vector space $V$. (Vector spaces, Lie algebras and modules shall be finite dimensional throughout this paper.) Whenever 9 is explicitly regarded as a $g$-module, we use the adjoint representation.

Definition. If $A$ is a subset of $g$ and $\lambda$ is a function from $A$ into $k, V_{A}^{\lambda}$ is the set of $x \in g$ such that some power of $\pi(a)-\lambda(a)$ annihilates $x$ for all $a \in A$. If $A=\{a\}$, write $V_{a}^{\lambda(a)}$ for $V_{A}^{\lambda}$. The functions $\lambda$ such that $V_{A}^{\lambda} \neq 0$ are weights, and any nonzero element of $V_{A}^{\lambda}$ is called a weight vector for $\lambda$. The set of weights will be denoted by $\Delta(V)$ or $\Delta_{A}(V)$.

Definition. A subset $A$ of $g$ is nil if $A \subset g_{A}^{0}$. (According to our convention, $\pi$ is the adjoint representation here.)

Received by the editors January 14, 1975.

AMS (MOS) subject classifications (1970). Primary 17B05; Secondary 17B20.

Key words and phrases. Cartan subspaces, symmetric Lie algebras, weight theory for nil sets, nil subspaces, natural $p$-subalgebras, splitting Cartan subspaces, reductive symmetric Lie algebras.

(1) Partially supported by NSF GP 33893. 
Definition. A linear operator on a vector space over $k$ is split if all its eigenvalues lie in $k$.

Proposition 1.1. Let $A$ be a nil subset of 9. Then:

(1) For all functions $\lambda: A \rightarrow k, V_{A}^{\lambda}$ is a subspace.

(2) For all functions $\lambda, \mu: A \rightarrow k,\left[g_{A}^{\lambda}, g_{A}^{\mu}\right] \subset g_{A}^{\lambda+\mu}$ and $g_{A}^{\lambda} \cdot V_{A}^{\mu} \subset$ $V_{A}^{\lambda+\mu}$.

(3) $g_{A}^{0}$ is a subalgebra of 9 , and $g_{A}^{\lambda}$ and $V_{A}^{\lambda}$ are invariant under $g_{A}^{0}$ and hence under $A$, for all functions $\lambda: A \rightarrow k$.

(4) $\Sigma_{\lambda \in \Delta(V)} V_{A}^{\lambda}$ is a direct sum.

(5) $\Delta(V)$ is finite.

(6) If $\pi(a)$ is split for all $a \in A$, then $V=\amalg_{\lambda \in \Delta(V)} V_{A}^{\lambda}$.

The standard proofs of the weight space decomposition theorem for nilpotent Lie algebras easily generalize to cover Proposition 1.1 (cf. [5, $\S \S 2.4$ and 2.1]).

G. Seligman has given an example where weights with respect to a nilpotent Lie algebra (and, in particular, a nil subspace) are not linear [6, Chapter V, §1]. But we have the following:

Proposition 1.2. Let a be a nil subspace of $g, \lambda \in \Delta_{a}(V)$ and $\operatorname{dim} V_{a}^{\lambda}=$ n. Then:

(1) $\lambda^{n}$ is a polynomial function on a.

(2) If char $k=0$, or more generally, if char $k$ does not divide $n$, then $\lambda$ is linear.

Proof. (1) For $x \in a, \lambda^{n}(x)$ is simply the determinant of the action of $\pi(x)$ on $V_{a}^{\lambda}$. (Strictly upper triangularize $\pi(x)-\lambda(x)$ on $V_{a}^{\lambda}$.)

(2) For $x \in a$, the trace of the action of $\pi(x)$ on $V_{a}^{\lambda}$ is just $n \lambda(x)$, so that $\lambda(x)=n^{-1} \operatorname{tr}\left(\pi(x) \mid V_{a}^{\lambda}\right)$. (Cf. the discussion on the linearity of weights after Corollary 1 to Theorem 4.2.)

If $W$ is any vector space over $k$ and $K$ is any extension of $k$, we denote by $W_{K}$ the extension of $W$ to a vector space over $K$; similarly for the Lie algebra $g$, the module $V$, the representation $\pi$, etc.

THEOREM 1.3. Let $k$ be infinite and $K$ an extension of $k$. If $a$ is $a$ subspace of 9 , we have:

(1) $\left(V_{a}^{0}\right)_{K}=\left(V_{K}\right)_{a_{K}}^{0}$.

(2) $a$ is nil if and only if $a_{K}$ is nil.

Proof. (1) If $x \in\left(V_{K}\right)_{a_{K}}^{0}$, express $x$ as $\Sigma k_{i} \otimes x_{i}$, where the $k_{i}$ are in $K$ and linearly independent over $k$, and the $x_{i}$ are in $V$. If we apply $\pi_{K}(a)$ repeatedly to $x$ for any $a \in a$, we find that each $x_{i} \in V_{\text {,a }}^{0}$, so that $x \in\left(V_{a}^{0}\right)_{K}$. Hence $\left(V_{a}^{0}\right)_{K}$ 
$\supset\left(V_{K}\right)_{a_{K}}^{0}$. Assume now that $\left(V_{a}^{0}\right)_{K} \neq\left(V_{K}\right)_{a_{K}}^{0}$, and let $n=\operatorname{dim} V$. Then there exists $b \in a_{K}$ such that $\pi_{K}(b)^{n}\left(V_{a}^{0}\right)_{K} \neq 0$. Choose a $K$-basis $v_{1}, \ldots, v_{m}$ of $\left(V_{a}^{0}\right)_{K}$, extend it to a basis $v_{1}, \ldots, v_{n}$ of $V_{K}$, and let $v_{i}^{*}, \ldots, v_{n}^{*}$ be the corresponding dual basis. Then $v_{i}^{*}\left(\pi_{K}(b)^{n} v_{j}\right) \neq 0$ for some $i=1, \ldots, n$ and $j=$ $1, \ldots, m$, and so $v_{i}^{*}\left(\pi_{K}(x)^{n} v_{j}\right)$ is a nonzero polynomial function on $a_{K}$, as $x$ varies. But $a$ is Zariski dense in $a_{K}$ since $k$ is infinite, so that the polynomial function does not vanish on $a$. Hence there exists $c \in a$ such that $\pi_{K}(c)^{n}\left(V_{a}^{0}\right)_{K}$ $\neq 0$. It follows that $\pi(c)^{n} V_{a}^{0} \neq 0$, and so $V_{a}^{0}$ contains an element not annihilated by any power of $\pi(c)$, contradicting the definition of $V_{a}^{0}$. (Note that it is not necessary for $\pi(a)$ to preserve $V_{a}^{0}$ in this proof.)

(2) From (1) it follows that if $a$ is nil, then $a_{K} \subset\left(g_{K}\right)_{a_{K}}^{0}$, so that $a_{K}$ is nil. The other direction is trivial.

TheOREM 1.4. Let $k$ be infinite, and let $A$ be a nil subset of 9 . Then there is an element $x$ in the linear span of $A$ such that $\pi(x)$ induces a nonsingular linear transformation on $V / V_{A}^{0}$.

Proof. Let $K$ be an algebraically closed field containing $k$, and let $a$ be the $k$-span of $A$ in $g$, so that $a_{K}$ is the $K$-span of $A$ in $g_{K}$. If we can find $b \in$ $a_{K}$ such that $\pi_{K}(b)$ induces a nonsingular linear transformation on $V_{K} /\left(V_{K}\right)_{A}^{0}=$ $\left(V / V_{A}^{0}\right)_{K}$, then the determinant of the action of $y$ on this space gives a nonzero polynomial function on $a_{K}$. Since $k$ is infinite, this function cannot vanish on $a$, and so we can find an element $c \in a$ such that $\pi_{K}(c)$ induces a nonsingular linear transformation on $\left(V / V_{A}^{0}\right)_{K}$. Hence $\pi(c)$ induces a nonsingular linear transformation on $V / V_{A}^{0}$, and we have reduced the theorem to the case in which $k$ is algebraically closed.

Assume that $k$ is algebraically closed, apply Proposition 1.1(6), and let $\lambda \in$ $\Delta_{A}(V), \lambda \neq 0$. Then since each $\pi(a)(a \in A)$ can be upper triangularized on $V_{A}^{\lambda}$ with diagonal entries all $\lambda(a)$, at least one $\pi(a)$ is nonsingular on $V_{A}^{\lambda}$. Thus $d_{\lambda}(a)$ $=\operatorname{det}\left(\pi(a) \mid V_{A}^{\lambda}\right)$ defines a nonzero polynomial function on the span a of $A$.

Since $k$ is infinite, $d=\Pi d_{\lambda}(\lambda \in \Delta(V), \lambda \neq 0)$ is a nonzero polynomial function on $a$. Clearly any $x \in a$ such that $d(x) \neq 0$ satisfies the requirements of the theorem.

We now comment briefly on the Fitting decomposition of $V$ (or $g$ ) with respect to a nil subset of $g$; we shall not need this for our main results.

Recall that the Fitting lemma says that if $F$ is a linear transformation on a vector space $W$, then $W=W_{F}^{0} \oplus W_{F}^{1}$, where $F$ is nilpotent on $W_{F}^{0}$ and nonsingular on $W_{F}^{1}$.

Definition. If $A$ is a nil subset of $g$, then $V_{A}^{1}$ is the sum of the Fitting components $V_{\pi(a)}^{1}$ for $a \in A$. If $A=\{a\}$, write $V_{a}^{1}$ for $V_{A}^{1}$; there should be no 
confusion with the notation introduced in the first definition of $\S 1$.

Proposition 1.5. Let $A$ be a nil subset of 9 . Then $V=V_{A}^{0} \oplus V_{A}^{1}$, and $V_{A}^{1}$ is the unique A-invariant complement of $V_{A}^{0}$.

Proof. Theorem 4 of [3, Chapter II] can easily be generalized to nil sets of linear operators, and then it gives the proposition when applied to $\{\pi(a) \mid a \in A\}$.

REMARKS. (1) If $A$ is a nil subset of $g$ and $\pi(a)$ is split for all $a \in A$, then $V_{A}^{1}=\amalg V_{A}^{\lambda}$, as $\lambda$ ranges through $\Delta_{A}(V)-\{0\} \quad$ (cf. Proposition 1.1(6)).

(2) In the context of Theorem 1.3, if $a$ is a nil subspace of $g$, then $\left(V_{a}^{1}\right)_{K}$ $=\left(V_{K}\right)_{a_{K}}^{1} ;$ this follows easily from Theorem 1.3 and Proposition 1.5.

(3) In the context of Theorem $1.4, \pi(x)$ is nonsingular on $V_{A}^{1}$.

(4) Theorem 5 of [3, Chapter II] can easily be extended by the arguments used there to nil sets.

2. Symmetric Lie algebras.

Definition. A symmetric Lie algebra over a field $k$ is a triple $(g ; E, p)$ such that $g$ is a Lie algebra over $k$ with subspaces $\xi$ and $p, g=\xi \oplus p,[\xi, \xi] \subset$ $E,[E, p] \subset p$ and $[p, p] \subset E$. We shall often denote the symmetric Lie algebra $(g ; E, p)$ simply by $g$, and we call $g=\notin \oplus p$ the symmetric decomposition of $g$. Let $\theta$ be the linear automorphism of $g$ which is 1 on $E$ and -1 on $p$. Then $\theta$ is called the symmetry of $\mathrm{g}$.

EXAMPLES. The most familiar example is that of a real semisimple Lie algebra $g$ with Cartan decomposition $g=\notin \oplus p$ in the classical sense that the Killing form of $g$ is negative definite on and positive definite on $p$. More generally, we have the "orthogonal symmetric Lie algebras" of E. Cartan (see [2, Chapter V, $\S \S 1,2])$. We get many other examples from Lie algebras $g$ graded by the integers (i.e., $g=\amalg g_{i}\left(i\right.$ an integer) with $\left[g_{i}, g_{j}\right] \subset g_{i+j}$ for all $\left.i, j\right)$; take $E=\amalg g_{i}$ ( $i$ even) and $p=\amalg g_{i}$ ( $i$ odd). One situation of this type is the grading by rootlevel of a split semisimple Lie algebra over characteristic zero.

The following result is immediate:

Proposition 2.1. Let $(g ; k, \uparrow)$ be a symmetric Lie algebra, with symmetry $\theta$. Then:

(1) is a subalgebra and $p$ is a

(2) $\theta$ is an automorphism of $g$ such that $\theta^{2}=1$.

(3) If char $k \neq 2$ and $\theta$ is an automorphism of 9 such that $\theta^{2}=1$, then the +1 and -1 eigenspaces of $\theta$ determine a symmetric Lie algebra structure on $\mathrm{g}$, and this sets up a bijection between the symmetric Lie algebra structures and such automorphisms, when char $k \neq 2$.

In view of (3), if char $k \neq 2$, we can denote symmetric Lie algebras by $(g, \theta)$, as well as by $(g ; \varepsilon, p)$. 
Proposition 2.2. If $(g ; €, p)$ is a symmetric Lie algebra, then $\notin$ and $p$ are orthogonal with respect to the Killing form of $\mathrm{g}$.

Proof. If $x \in \mathcal{E}$ and $y \in p$, then ad $x$ ad $y$ maps $E$ into $p$ and $p$ into $E$, and hence is traceless.

Definition. If $A$ is a subset of the symmetric Lie algebra $g$, then $p_{A}^{0}=p$ $\cap g_{A}^{0}, p_{A}^{1}=p \cap g_{A}^{1}, E_{A}^{0}=\notin \cap g_{A}^{0}$ and $\mathfrak{E}_{A}^{1}=\notin \cap g_{A}^{1}$. If $A=\{a\}$, write $\vee_{a}^{0}$, etc.

Proposition 2.3. Let $A$ be a nil subset of $p$. Then $g_{A}^{0}=\varepsilon_{A}^{0} \oplus p_{A}^{0}$, $g_{A}^{1}=E_{A}^{1} \oplus \mathfrak{p}_{A}^{1}, \mathfrak{p}=\mathfrak{p}_{A}^{0} \oplus \mathrm{F}_{A}^{1}, \mathfrak{t}=\mathfrak{E}_{A}^{0} \oplus \mathfrak{E}_{A}^{1}$, and $\mathrm{g}=\mathfrak{E}_{A}^{0} \oplus \mathcal{E}_{A}^{1} \oplus \mathfrak{F}_{A}^{0} \oplus \mathrm{p}_{A}^{1}$.

Proof. The last three equations follow easily from the first two, and it is easy to reduce each of these to the case when $A$ has only one element. But if $x \in p$, then $g_{x}^{0}=\left\{y \in g \mid(\operatorname{ad} x)^{n} y=0\right.$ for some $\left.n\right\}$, and because ad $x$ switches the subspaces $E$ and $p$ back and forth, $(\operatorname{ad} x)^{n} y=0$ for some $n$ if and only if some power of ad $x$ kills each component of $y$ with respect to the decomposition $g=€ \oplus p$. Similarly, $g_{x}^{1}$ is the intersection of all the images of $(\operatorname{ad} x)^{n}$, and it suffices to take even powers. But $(\operatorname{ad} x)^{2 n} g=(\operatorname{ad} x)^{2 n} \mathfrak{E} \oplus(\operatorname{ad} x)^{2 n} p$, and so the result follows.

REMARK. A similar result holds for a nil subset $A$ of $\varepsilon$.

3. Cartan subspaces and natural $p$-subalgebras. This section contains the main existence and characterization theorems for Cartan subspaces. Slightly more refined results for characteristic zero and for $g$ reductive will be obtained in $\S \S 4$ and 5.

From now on, the field $k$ will be assumed infinite.

Definition. A p-subalgebra of the symmetric Lie algebra $g$ is a subspace $F_{1}$ of $p$ such that for all $x \in p_{1},(\operatorname{ad} x)^{2} p_{1} \subset p_{1}$.

Definition. $A p$-subalgebra $p_{1}$ is natural if there exists $x_{0} \in p_{1}$ such that $\left(\operatorname{ad} x_{0}\right)^{2}$ induces a nonsingular linear transformation on $p / p_{1}$.

Note that $p$ itself is a natural $p$-subalgebra. Theorem 3.1 provides many others.

THEOREM 3.1. If $A$ is a nil subset of $p$, then $p_{A}^{0}$ is a natural $p$-subalgebra, and the required element may be chosen from the span of $A$. In particular, if a is a nil subspace of $F$, then the required element may be chosen from a.

PROOF. Since $g_{A}^{0}$ is a subalgebra and hence is certainly preserved by $(\operatorname{ad} x)^{2}$ for any $x \in p_{A}^{0}$, and since $p$ is also preserved by $(\operatorname{ad} x)^{2}$ for such $x, p_{A}^{0}$ is a $p$-subalgebra. By Theorem 1.4, there is an element $x_{0}$ in the span of $A$ such that ad $x_{0}$ induces a nonsingular linear transformation on $g / g_{A}^{0}$. Hence its square is certainly nonsingular on $\mathrm{p} / \mathrm{p}_{A}^{0}$. But $x_{0} \in \mathfrak{p}_{A}^{0}$ since $x_{0}$ is in the span of $A$. 
Definition. A Cartan subspace of the symmetric Lie algebra $g$ (or a Cartan subspace of $p$ ) is a subset $a$ of $p$ such that $a=p_{a}^{0}$. Clearly $a$ is a nil subspace of $p$.

REMARK. If $g$ is an ordinary Lie algebra (over any field), a subset $\bar{g}$ of $g$ is a Cartan subalgebra if and only if $h=g_{\mathfrak{h}}^{0}$.

DEFINITION. An element $x$ of $p$ is $p$-generic if $\operatorname{dim} p_{x}^{0}$ is as small as possisible.

REMARK. The set of $p$-generic elements is the set of nonzeros of a polynomial function on $\downarrow$. In fact, for $x \in p, p_{x}^{0}$ is the generalized zero eigenspace of the endomorphism $(\operatorname{ad} x)^{2} \mid p$ of $p$. Just take the appropriate coefficient of the characteristic polynomial of this endomorphism.

THEOREM 3.2. Let a be a subset of $₹$. Then the following are equivalent:

(i) $a$ is a Cartan subspace.

(ii) a is a minimal natural $p$-subalgebra.

(iii) $a=p_{x}^{0}$ for some $x \in p$ and $a$ is nil.

(iv) $a$. is a nil natural $p$-subalgebra.

Proof. (i) $\Rightarrow$ (ii). If $a$ is a Cartan subspace, it is a natural $p$-subalgebra by Theorem 3.1. Now suppose that $p_{1} \subset a$ is a natural $p$-subalgebra, and let $x_{0} \in$ $p_{1}$ be such that $\left(\operatorname{ad} x_{0}\right)^{2}$ induces a nonsingular linear transformation on $p / p_{1}$. Then $\left(\operatorname{ad} x_{0}\right)^{2}$ induces a linear transformation on $a / p_{1}$ which is both nonsingular and nilpotent, so that $a=p_{1}$.

(ii) $\Rightarrow$ (iii). Let $d$ be the polynomial function on $a$ whose value at $x \in a$ is the determinant of $(\operatorname{ad} x)^{2}$ on $p / a$. Choose $x_{0} \in a$ such that $d\left(x_{0}\right) \neq 0$. Then $p_{x_{0}}^{0} \subset a$ because $\left(\operatorname{ad} x_{0}\right)^{2}$ induces a nonsingular linear transformation on $p / a$. But $p_{x_{0}}^{0}$ is natural by Theorem 3.1. Hence $a=p_{x_{0}}^{0}$. Let $n=\operatorname{dim} a$. If $a$ is not nil, there exists $x \in a$ such that $(\operatorname{ad} x)^{2 n} \mid a \neq 0$. Then some matrix entry of $(\operatorname{ad} x)^{2 n} \mid a$ with respect to a basis of a must be nonzero. This matrix entry defines a nonzero polynomial function $f$ on $a$. Since $k$ is infinite, $f d$ is a nonzero polynomial function on $a$, and so we may choose $y \in a$ such that $(f d)(y) \neq 0$. But then $(\operatorname{ad} y)^{2 n} \mid a \neq 0$, so that $(\operatorname{ad} y)^{2}$ is not nilpotent on $a$. Then $p_{y}^{0}$ is a strictly smaller natural $p$-subalgebra, which is impossible.

(iii) $\Rightarrow$ (iv). This follows immediately from Theorem 3.1.

(iv) $\Rightarrow$ (i). Since $a$ is nil, $a \subset p_{a}^{0}$. But it is also equal since it is natural.

Corollary 1. Cartan subspaces exist.

Proof. Since $\not$ is a natural $p$-subalgebra, minimal natural $\not$-subalgebras exist.

COROLlaRY 2. If $x \in \emptyset$ is $p$-generic, then $\vDash_{x}^{0}$ is a Cartan subspace. 
PROof. If not, it must contain a natural $p$-subalgebra $F_{1}$ properly. Choose $x_{0} \in p_{1}$ such that $\left(\operatorname{ad} x_{0}\right)^{2}$ induces a nonsingular linear transformation on $p / p_{1}$. Then $p_{x_{0}}^{0} \subset p_{1}$, which is properly contained in $p_{x}^{0}$, contradicting the assumption that $x$ is $p$-generic.

THEOREM 3.3. Let $p_{1}$ be a $p$-subalgebra. Then the following are equivalent:

(i) $p_{1}$ is natural.

(ii) $P_{1}$ contains a natural $p$-subalgebra.

(iii) $F_{1}$ contains a Cartan subspace.

(iv) $F_{1}$ contains $p_{x}^{0}$ for some $x \in p$.

Proof. (i) $\Rightarrow$ (ii) is obvious.

(ii) $\Rightarrow$ (iii). Choose a minimal natural $f$-subalgebra contained in $p_{1}$.

(iii) $\Rightarrow$ (iv) is clear since any Cartan subspace is of this form.

(iv) $\Rightarrow$ (i). If $p_{1}$ contains $p_{x}^{0}$, then $(\operatorname{ad} x)^{2}$ acts nonsingularly on $p / p_{x}^{0}$, so it certainly acts nonsingularly on $f / p_{1}$.

THEOREM 3.4. Let $K$ be an extension of $k$ and a a subspace of $p$. Then:

(1) $a$ is $a p$-subalgebra if and only if $a_{K}$ is a $p$-subalgebra (of the symmetric Lie algebra $\left.\left(g_{K} ; \mathfrak{k}_{K}, \mathfrak{p}_{K}\right)\right)$. (This holds even if $k$ is finite.)

(2) $a$ is a natural $p$-subalgebra if and only if $a_{K}$ is.

(3) $a$ is a Cartan subspace if and only if $a_{K}$ is.

Proof. (1) Suppose $a$ is a $p$-subalgebra. Then for all $x, y \in a$, $\operatorname{ad} x$ ad $y+\operatorname{ad} y$ ad $x=(\operatorname{ad}(x+y))^{2}-(\operatorname{ad} x)^{2}-(\operatorname{ad} y)^{2}$

preserves $a$. A general element of $a_{K}$ is of the form $\Sigma_{i=1}^{r} k_{i} \otimes a_{i}$, with $r \geqslant 0$, $k_{i} \in K$ and $a_{i} \in a$. Then $a_{K}$ is a $p$-subalgebra since

$$
\left(\operatorname{ad}\left(\sum_{i=1}^{r} k_{i} \otimes a_{i}\right)\right)^{2}=\sum_{i=1}^{r} k_{i}^{2}\left(\operatorname{ad} a_{i}\right)^{2}+\sum_{i<j} k_{i} k_{j}\left(\operatorname{ad} a_{i} \operatorname{ad} a_{j .}+\operatorname{ad} a_{j} \operatorname{ad} a_{i}\right) .
$$

The other direction is clear.

(2) If $a$ is natural, choose $x \in a$ such that ( $\operatorname{ad} x)^{2}$ induces a nonsingular linear transformation on $\mathrm{p} / \mathrm{a}$. Then $(\mathrm{ad} x)^{2}$ also induces a nonsingular linear transformation on $p_{K} / a_{K}$, so $a_{K}$ is natural. Now assume that $a_{K}$ is natural. Then the determinant of the linear transformation induced on $p_{K} / a_{K}$ by $(\mathrm{ad} x)^{2}$ $\left(x \in a_{K}\right)$ is a nonzero polynomial function on $a_{K}$. Its restriction to $a$ must also then be nonzero. Thus there exists $x \in a$ such that $(\mathrm{ad} x)^{2}$ induces a nonsingular linear transformation on $p_{K} / a_{K}$, and hence also on $p / a$. 
(3) By Theorem 1.3(1), $\left(p_{a}^{0}\right)_{K}=\left(p_{K}\right)_{a}^{0}$ for any subspace a of $g$. Now just apply the definition of Cartan subspace.

Definition. An element $x \in g$ is semisimple if ad $x$ is a semisimple linear transformation of $\mathrm{g}$.

THEOREM 3.5. Let $S$ be a pairwise commuting family of semisimple elements of $\not$. Then $S$ is a subset of some Cartan subspace.

Proof. $S$ is clearly nil, so by Theorem 3.1,$\urcorner_{S}^{0}$ is a natural $\downarrow$-subalgebra. Since the elements of $S$ are semisimple, $p_{S}^{0}$ is in fact the centralizer of $S$ in $p$. Now choose a minimal natural $p$-subalgebra a contained in $p_{S}^{0}$. Then $a$ is a Car$\tan$ subspace by Theorem 3.2. But a centralizes $S$, and so $S \subset p_{a}^{0}=x$. (Note that all we have really used about the elements of $S$ is that $p_{s}^{0}$ is contained in the centralizer of $s$ for all $s \in S$.)

4. The characteristic zero case. From now on, char $k=0$. First we show that when $k$ is algebraically closed, all Cartan subspaces of the symmetric Lie algebra $g$ are conjugate under the algebraic group associated with $\mathfrak{E}$. If $k$ is not assumed algebraically closed, then all Cartan subspaces still have the same dimension. One can thus define the rank of a symmetric Lie algebra to be this common dimension. We also present a result of $D$. Haile that the subalgebra generated by a Cartan subspace is nilpotent.

THEOREM 4.1. Assume that $k$ is algebraically closed, and denote by $G$ the smallest algebraic subgroup of Aut $g$ whose Lie algebra contains $\operatorname{ad}_{g} E$ (using the obvious notation). Then all Cartan subspaces of $p$ are conjugate under $G$. If $k$ is the field of complex numbers, then all Cartan subspaces of $p$ are even conjugate under the Lie subgroup of Aut $g$ whose Lie algebra is $\operatorname{ad}_{g} \xi$.

Proof. The proof of the last assertion is similar to the proof of conjugacy of Cartan subalgebras given in [7, p. 263, Theorem 4.1.3]. To prove the first assertion, first note that $G$ preserves $p$, since the set of automorphisms of 9 commuting with the symmetry $\theta$ of $g$ is an algebraic group. Let $a_{1}, a_{2}$ be Cartan subspaces, and for $i=1,2$, let $U_{i}=\left\{x \in a_{i} \mid(\operatorname{ad} x)^{2}\right.$ induces a nonsingular linear transformation on $\left.p / a_{i}\right\}$. Each $U_{i}$ is open, and if $x \in U_{i}$, then $a_{i}=p_{x}^{0}$. Now consider the natural map $\varphi_{i}: G \times U_{i} \rightarrow p$. If $x \in U_{i}$, the image of the differential of $\varphi_{i}$ at $(1, x)$ contains $a_{i}$, since $U_{i}$ is open. But it also contains $[\mathcal{E}, x]$ and hence $(\operatorname{ad} x)^{2} p$. Since $p=a_{i}+(\operatorname{ad} x)^{2} p$, the differential maps onto $k$, and so the image of $\varphi_{i}$ has nonempty interior in $p$. Since $p$ is irreducible, we may choose $x_{0} \in \operatorname{Im} \varphi_{1} \cap \operatorname{Im} \varphi_{2}, g_{i} \in G$ and $x_{i} \in U_{i}$ such that $g_{i}\left(x_{i}\right)=x_{0}(i=1,2)$. But then $\left(g_{1}^{-1} g_{2}\right)\left(x_{2}\right)=x_{1}$, and $g_{1}^{-1} g_{2}$ conjugates $a_{2}$ to $a_{1}$. 
Corollary 1. All Cartan subspaces have the same dimension.

Proof. First extend scalars to the algebraic closure, and then apply Theorems 3.4 (3) and 4.1.

Definition. The rank of $(g ; \mathfrak{k}, k)$ is the common dimension of its Cartan subspaces.

COROLlary 2. All Cartan subspaces are of the form $\wp_{x}^{0}$ where $x$ is $p-g e$ neric.

Proof. If $a$ is a Cartan subspace, then $a=F_{x}^{0}$ for some $x \in a$, by Theorem 3.2. If $y$ is any $p$-generic element, then $p_{y}^{0}$ is also a Cartan subspace (Corollary 2 of Theorem 3.2). But it must have the same dimension as $a$, so that $x$ is also $p$-generic.

THEOREM 4.2 (HAILE). The subalgebra generated by a Cartan subspace is nilpotent.

Proof. Let $g_{0}=a \oplus[a, a]$. We first show that $g_{0}$ is the subalgebra generated by $a$. But $[a,[a, a]] \subset 9_{a}^{0} \cap p=a$. Thus $[[a, a],[a, a]] \subset$ $[a, a]$, and so $g_{0}$ is indeed a subalgebra, and a clearly generates it.

Next, $\left(g_{0} ;[a, a], a\right)$ is a symmetric Lie algebra. By Proposition 2.2, a and $[a, a]$ are orthogonal with respect to the Killing from $B_{0}$ of $g_{0}$. Also, for all $x \in a,(\operatorname{ad} x)^{2}$ is nilpotent on $g_{0}$, since $g_{0} \subset g_{a}^{0}$. Thus $B_{0} \mid a$ is both alternating and symmetric, and hence zero. Thus $a \subset \operatorname{rad} B_{0}$, so that $a \subset \operatorname{rad} g_{0}$, and $g_{0}$ is solvable. But it follows from Lie's theorem that in a solvable Lie algebra, the elements $x$ such that ad $x$ is nilpotent form an ideal (cf. [5, Exercise 4 for Part 2]). Since $(\operatorname{ad} x) \mid g_{0}$ is nilpotent for all $x \in a$, the same must be true for all $x \in g_{0}$, and so $g_{0}$ is nilpotent by Engel's theorem.

Corollary 1. Let a be a Cartan subspace and let $\pi: g \rightarrow$ End $V$ be $a$ representation such that $\pi(a)$ is split for all $a \in a$. Then the $\pi(a)(a \in a)$ can be simultaneously upper triangularized on each weight space $V_{a}^{\lambda}\left(\lambda \in \Delta_{a}(V)\right)$, with diagonal matrix entries all $\lambda(a)$.

Proof. Let $g_{0}$ be the subalgebra generated by $a$. Then $g_{0}$ is solvable by the theorem. Since $g_{0} \subset g_{a}^{0}, \pi\left(g_{0}\right)$ preserves each $V_{a}^{\lambda}$. But Lie's theorem implies that if a solvable Lie algebra of linear operators (over $k$ ) is generated by a set of split operators, then it can be simultaneously upper triangularized. (Indeed, extend to the algebraic closure $K$ of $k$, apply Lie's theorem over $K$, use this to show that the original Lie algebra over $k$ consists of split operators, and then apply Lie's theorem over $k$.) The corollary now follows.

This corollary leads to the usual proof that weights are linear, as in [5, Theorem 13 of $\S 2.1$ and Theorem 3 of $\S 2.4$ ]. But recall that Proposition 1.2(2) 
shows the linearity of weights much more generally.

REMARK. Using Lie's theorem as in the proof of the corollary, it is easy to see that if $A$ is a nil subset of a Lie algebra $g$ over $k$ such that ad $a$ is split for all $a \in A$, then the following are equivalent:

(i) ad $A$ can be simultaneously upper triangularized.

(ii) The subalgebra of $g$ generated by $A$ is solvable.

(iii) The subalgebra of $g$ generated by $A$ is nilpotent.

(iv) On $g_{A}^{0}$, ad $A$ can be simultaneously strictly upper triangularized.

Definition. A splitting Cartan subspace of $g$ (or of $p$ ) is a Cartan subspace $a$ of $g$ such that for all $a \in a$, the linear transformation ad $a$ of $g$ is split.

Theorems 3.4(3) and 4.2 and the last Remark imply:

Corollary 2. Let $K$ be an extension of $k$ and a a splitting Cartan subspace of $g$. Then $a_{K}$ is a splitting Cartan subspace of $g_{K}$.

Remark. A nil set need not span a nil subspace. Example: $\left(\begin{array}{ll}0 & 1 \\ 0 & 0\end{array}\right)$ and $\left(\begin{array}{ll}0 & 0 \\ 1 & 0\end{array}\right)$ in $\& \mathfrak{L}(2)$. Also, a nil subspace need not generate a nilpotent subalgebra (but cf. Theorem 4.2). Example: The span of

$$
\left(\begin{array}{lll}
0 & 1 & 0 \\
0 & 0 & -1 \\
0 & 0 & 0
\end{array}\right) \text { and }\left(\begin{array}{lll}
0 & 0 & 0 \\
1 & 0 & 0 \\
0 & 1 & 0
\end{array}\right)
$$

in $\dot{s}$ L (3) (cf. Amer. Math. Monthly 81 (1974), 913, Solution of Problem 5921, proposed by Paul Cohn).

5. Reductive symmetric Lie algebras in characteristic zero. In this section, we show that any Cartan subspace of a reductive symmetric Lie algebra can be embedded in a Cartan subalgebra. We also show that our definition of Cartan sub space agrees with the classical definition (see $[1,1.13]$ ) in this case.

THEOREM 5.1. Let $(g ; \xi, p)$ be a reductive symmetric Lie algebra, a a Cartan subspace, and $m$ the centralizer of $a$ in $\xi$. Then $\xi$ and $m$ are reductive Lie algebras, $a$ is an abelian Lie algebra contained in the center of $g_{a}^{0}$, and $\left(g_{a}^{0}\right.$; $\mathfrak{m}, a)$ is a reductive symmetric Lie algebra.

Proof. Let $\theta$ be the symmetry of $g$. Then $\theta$ preserves the center of $g$ and $[g, g]$. It follows easily that all the spaces mentioned in the theorem are the sums of their intersections with the center of $g$ and with $[g, g]$, and we may thus pass to the case in which $g$ is semisimple. Since the Killing form $B$ of $g$ is a nonsingular trace form on $E, E$ is reductive (see either $[5, \S 2.9]$ or $[1,1.7 .3]$ ). For the other statements, we may pass to the algebraic closure of $k$. Then $g$ splits into a direct sum of weight spaces under the action of $a$, and if $\lambda$ and $\mu$ are weights, then $B\left(g_{a}^{\lambda}, g_{a}^{\mu}\right)=0$ if $\lambda \neq-\mu$. Thus $B\left(g_{a}^{0}, g_{a}^{\lambda}\right)=0$ for all $\lambda \neq 0$, and 
so $B$ gives a nonsingular trace form on $g_{a}^{0}$. Hence $g_{a}^{0}$ is reductive. Just as in the proof of Theorem 4.2, $a$ is contained in the radical of the Killing form of $g_{a}^{0}$, hence in the radical of $g_{a}^{0}$. Thus $a$ is abelian, and is contained in the center of $g_{a}^{0}$. It now follows that $\mathfrak{E}_{a}^{0}$ must equal $m$, and $g_{a}^{0}=\mathfrak{m} \oplus a$ by Proposition 2.3. Hence $\left(g_{a}^{0} ; m, a\right)$ is a reductive symmetric Lie algebra. Finally, since $B(m, a)=0, B$ must be nonsingular on $m$, and so $m$ is reductive.

REMARK (D. HAILE). Cartan subspaces need not be abelian for nonreductive symmetric Lie algebras $g$. In fact, let $g$ be the Lie algebra of $n \times n$ upper triangular matrices, make $g$ a graded Lie algebra by taking $g_{i}$ to be the $i$ th diagonal above the main diagonal $(i \geqslant 0)$, and let $\xi$ and $k$ be the even and odd subspaces, as in the Examples at the beginning of $\S 2$. Then $p$ is a nonabelian Cartan subspace of itself, as long as $n \geqslant 3$.

THEOREM 5.2. Let a be a Cartan subspace of a reductive symmetric Lie algebra 9 , and let $\mathrm{m}$ be the centralizer of $a$ in $\mathfrak{k}$. Choose a Cartan subalgebra $\mathfrak{l}$ of $\mathrm{m}$. Then $\mathfrak{l} \oplus$ a is a Cartan subalgebra of $\mathrm{g}$.

Proof. Since $\mathfrak{l}$ and $a$ are nilpotent Lie algebras and their elements commute, $\mathfrak{l} \oplus a$ is a nilpotent Lie algebra. Now any element of $g_{\mathfrak{l} \oplus a}^{0}$ must be in $g_{a}^{0}$, and so it is of the form $y+z$, with $y \in m$ and $z \in a$. But $y \in m_{\mathfrak{l}}^{0}$, and so $y \in \mathfrak{l}$. Hence $g_{\mathfrak{l} \oplus a}^{0}=\mathfrak{l} \oplus a$.

COROLlary. Let $(g ; k, p)$ be a reductive symmetric Lie algebra, and a a subset of $\mathrm{p}$. Then the following are equivalent:

(i) a is a Cartan subspace.

(ii) $a$ is a maximal abelian subspace of $p$ consisting of semisimple elements.

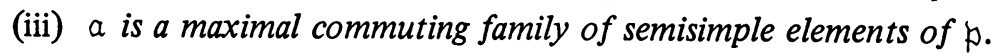

Proof. (i) $\Rightarrow$ (ii). The abelian subspace a consists of semisimple elements because it lies in a Cartan subalgebra of g. It is maximal abelian because $a=$ for.

(ii) $\Rightarrow$ (iii). If it were possible to find a bigger set of commuting semisimple elements in $p$, its span would be a bigger abelian subspace of $p$.

(iii) $\Rightarrow$ (i). a can be embedded in a Cartan subspace, by Theorem 3.5 , but it must be equal to this subspace, which is a commuting family of semisimple elements.

In a real semisimple Lie algebra $g$ with Cartan decomposition in the classical sense that the Killing form is negative definite on $E$ and positive definite on $p$, Cartan subspaces are often defined to be maximal abelian subspaces of $p$. But in this case, all elements of $p$ are semisimple, so that our definition agrees with the classical one. 
REMARK (HAILE). The reductivity cannot be dropped from Theorem 5.2, as demonstrated by the two-dimensional nonabelian Lie algebra $g$ spanned by $x$

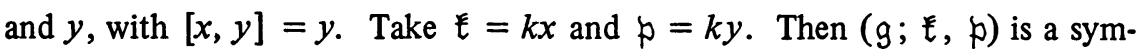
metric Lie algebra, $a=\mathfrak{p}$ is a Cartan subspace of $\mathfrak{p}$, and $m=\mathfrak{l}=0$. But $\mathfrak{l} \oplus$ $a=p$ is not a Cartan subalgebra of $g$, since $g=g_{k}^{0}$. It does not help to replace $m$ by $€_{a}^{0}$ in Theorem 5.2 (without reductivity), because in this example, $£_{a}^{0}=\mathfrak{E}$, with Cartan subalgebra $€$, and $€ \oplus a=g$ is not a Cartan subalgebra of itself. In fact, in this example, a cannot be extended to any Cartan subalgebra of $g$.

\section{REFERENCES}

1. J. Dixmier, Algèbres enveloppantes, Gauthier-Villars, Paris, 1974.

2. S. Helgason, Differential geometry and symmetric spaces, Pure and Appl Math., vol. 12, Academic Press, New York, 1962. MR 26 \#2986.

3. N. Jacobson, Lie algebras, Interscience Tracts in Pure and Appl. Math., no. 10, Interscience, New York, 1962. MR 26 \#1345.

4. B. Kostant and S. Rallis, Orbits and representations associated with symmetric spaces, Amer. J. Math. 93 (1971), 753-809. MR 47 \#399.

5. J. Lepowsky and G. W. McCollum, Elementary Lie algebra theory, Yale University Lecture Note Series, 1974, 138 pp.

6. G. B. Seligman, Modular Lie algebras, Ergebnisse der Mathematik und ihrer Grenzgebiete, Band 40, Springer-Verlag, New York, 1967. MR 39 \#6933.

7. V. S. Varadarajan, Lie groups, Lie algebras, and their representations, Prentice-Hall, Englewood Cliffs, N.J., 1974.

DEPARTMENT OF MATHEMATICS, YALE UNIVERSITY, NEW HAVEN, CONNECTICUT 06520

DEPARTMENT OF MATHEMATICS, TUFTS UNIVERSITY, MEDORD, MASSACHU. SETTS 02155 